\title{
ON THE DIGITS OF SQUARES AND THE DISTRIBUTION OF QUADRATIC SUBSEQUENCES OF DIGITAL SEQUENCES
}

\author{
ROSWITHA HOFER, GERHARD LARCHER, AND HEIDRUN ZELLINGER
}

(Communicated by Matthew A. Papanikolas)

\begin{abstract}
Let $q$ be a prime and $\gamma=\left(\gamma_{0}, \gamma_{1}, \gamma_{2}, \ldots\right)$ with $\gamma_{i} \in\{0, \ldots, q-1\}$ and $\gamma_{i}=0$ for $i \geq i_{0}$ be a weight sequence.

We study $\lim _{N \rightarrow \infty} \#\left\{0 \leq n<N \mid s_{q, \gamma}\left(n^{2}\right) \equiv d(\bmod q)\right\} / N$ with $s_{q, \gamma}\left(n^{2}\right)$ the weighted sum of digits of $n^{2}$ in base $q$ and we use the results to classify the digital sequences $\left(\boldsymbol{x}_{n}\right)_{n>0}$ in the sense of Niederreiter, generated by matrices with finite rows, for which $\left(\boldsymbol{x}_{n^{2}}\right)_{n>0}$ is uniformly distributed. Finally we derive an upper bound for the star discrepancy of these uniformly distributed subsequences.
\end{abstract}

\section{INTRODUCTION}

Let $S=\left(\boldsymbol{x}_{n}\right)_{n \geq 0}$ be a sequence of points in $[0,1)^{s}$. We say that $S$ is uniformly distributed modulo one if, for all subintervals $J$ of $[0,1)^{s}$, it is true that

$$
\lim _{N \rightarrow \infty} \frac{\#\left\{0 \leq n<N: \boldsymbol{x}_{n} \in J\right\}}{N}=\lambda(J),
$$

where $\lambda(J)$ denotes the Lebesgue measure of $J$.

We define the star discrepancy of the first $N$ points of the sequence $S$ by

$$
D_{N}^{*}(S):=\sup _{J \subseteq[0,1)^{s}}\left|\frac{\#\left\{0 \leq n<N: \boldsymbol{x}_{n} \in J\right\}}{N}-\lambda(J)\right|,
$$

where the supremum is extended over all intervals $J \subseteq[0,1)^{s}$ for which their lower left corner is in the origin.

The most basic and well-known uniformly distributed sequences in $[0,1)^{s}$ are the Kronecker sequences $\left(\boldsymbol{x}_{n}=\left(\left\{n \alpha_{1}\right\}, \ldots,\left\{n \alpha_{s}\right\}\right)\right)_{n \geq 0}$ with $1, \alpha_{1}, \ldots, \alpha_{s}$ linearly independent over $\mathbb{Q}$, the Halton sequences, and the digital $(t, s)$-sequences in the sense of Niederreiter Nie92 (or, more generally, $(\mathbf{T}, s)$-sequences in the sense of Larcher and Niederreiter; see LN95).

In various papers, subsequences of uniformly distributed sequences were considered and their distribution properties were studied. For Kronecker sequences this

Received by the editors April 26, 2011 and, in revised form, September 5, 2011.

2010 Mathematics Subject Classification. Primary 11K06, 11K31, 11K38.

Key words and phrases. Uniform distribution, discrepancy, digital $(\mathbf{T}, s)$-sequences, quadratic subsequences, digits of squares.

The first author was supported by the Austrian Science Fund under Project Nr. P21943.

The second author was supported by the Austrian Science Fund under Projects Nr. P21196 and P21943.

The third author is a recipient of a DOC-fForte Grant of the Austrian Academy of Sciences at the Institute of Financial Mathematics at the University of Linz (Austria). 
was done for example in [Vin48] or [Erd46] (for an overview, see [KN74] or [DT97]). For the Halton sequence this was done in HKLP09] and for digital $(\mathbf{T}, s)$-sequences (and more generally for Niederreiter-Halton sequences) in [HKLP09] and [Hof09]. In particular it is known that the quadratic subsequence $\left(\boldsymbol{x}_{n^{2}}\right)_{n>0}$ of a uniformly distributed Kronecker sequence $\left(\boldsymbol{x}_{n}\right)_{n>0}$ is again uniformly distributed. In contrast to this it is easily seen that the subsequence $\left(\boldsymbol{x}_{n^{2}}\right)_{n \geq 0}$ of a Halton sequence is never uniformly distributed.

In this paper we ask the question for which digital $(\mathbf{T}, s)$-sequences $\left(\boldsymbol{x}_{n}\right)_{n \geq 0}$ the subsequence $\left(\boldsymbol{x}_{n^{2}}\right)_{n \geq 0}$ is uniformly distributed.

A digital $(\mathbf{T}, s)$-sequence $\left(\boldsymbol{x}_{n}\right)_{n \geq 0}$ in $[0,1)^{s}$ is defined as follows.

Definition 1. Choose $s \mathbb{N}_{0} \times \mathbb{N}_{0}$ matrices $C^{(1)}, \ldots, C^{(s)}$ over $\mathbb{Z}_{q}, q$ prime. To generate the $i$ th coordinate $x_{n}^{(i)}$ of $\boldsymbol{x}_{n}$, represent the integer $n$ in base $q$ by

$$
n=n_{0}+n_{1} q+\cdots+n_{r} q^{r},
$$

set

$$
\boldsymbol{n}:=\left(n_{0}, \ldots, n_{r}, 0,0, \ldots\right)^{T}
$$

and compute

$$
C^{(i)} \cdot \boldsymbol{n}=:\left(y_{0}^{(i)}, y_{1}^{(i)}, \ldots\right)^{T}
$$

Finally, set

$$
x_{n}^{(i)}:=\frac{y_{0}^{(i)}}{q}+\frac{y_{1}^{(i)}}{q^{2}}+\ldots .
$$

Then $\left(\boldsymbol{x}_{n}\right)_{n \geq 0}$ is called a digital $(\mathbf{T}, s)$-sequence over $\mathbb{Z}_{q}$, where the parameter $\mathbf{T}$ : $\mathbb{N} \rightarrow \mathbb{N}_{0}$ is defined as follows. For every $m \in \mathbb{N}$, let $\mathbf{T}(m)$, satisfying $0 \leq \mathbf{T}(m) \leq$ $m$, be such that for all $d_{1}, d_{2}, \ldots, d_{s} \in \mathbb{N}_{0}$ with $d_{1}+\cdots+d_{s}=m-\mathbf{T}(m)$ the $(m-\mathbf{T}(m)) \times m$ matrix consisting of the

left upper $d_{1} \times m$ submatrix of $C^{(1)}$ together with the

left upper $d_{2} \times m$ submatrix of $C^{(2)}$ together with the

left upper $d_{s} \times m$ submatrix of $C^{(s)}$

has rank $m-\mathbf{T}(m)$. If $\mathbf{T}$ is minimal with this property, we speak of a strict digital ( $\mathbf{T}, s)$-sequence. If $\mathbf{T}(m) \leq t$ for all $m$, then we speak of a digital $(t, s)$-sequence. (Note that for the sake of simplicity we do not distinguish the residue classes of $\mathbb{Z}_{q}$ from their representatives $\{0,1, \ldots, q-1\}$.)

It is well known that a strict digital $(\mathbf{T}, s)$-sequence is uniformly distributed if and only if $\lim _{m \rightarrow \infty}(m-\mathbf{T}(m))=\infty$ or equivalently the rows of the generator matrices $C^{(1)}, \ldots, C^{(s)}$ altogether are linearly independent over $\mathbb{Z}_{q}$, i.e., that any finite set of rows of $C^{(1)}, \ldots, C^{(s)}$ is linearly independent over $\mathbb{Z}_{q}$.

As pointed out for example in [HL10, for the investigation of finer distribution properties of digital $(\mathbf{T}, s)$-sequences it makes a great difference if the generating matrices $C^{(1)}, \ldots, C^{(s)}$ consist of finite rows exclusively (i.e., for all $i \in\{1, \ldots, s\}$ and all $j \in\{0,1,2, \ldots\}$ we have $c_{j k}^{(i)} \neq 0$ for only finitely many $k$ ) or not.

In the latter case the investigations are often much more difficult.

In this paper we answer our question for digital $(\mathbf{T}, s)$-sequences generated by matrices with finite rows and we show the following main result. 
Theorem 1. Let $\left(\boldsymbol{x}_{n}\right)_{n>0}$ be a digital $(\mathbf{T}, s)$-sequence over $\mathbb{Z}_{q}$, q prime, generated by matrices $C^{(1)}, \ldots, C^{(s)}$ with finite rows. Then $\left(\boldsymbol{x}_{n^{2}}\right)_{n \geq 0}$ is uniformly distributed if and only if $\left(\boldsymbol{x}_{n}\right)_{n \geq 0}$ is uniformly distributed, $q=2$, and the sum of any finite set of rows of $C^{(1)}, \ldots, C^{(s)}$ is either of the form

$$
\left(\gamma_{0}, \gamma_{1}, \quad \gamma_{2}, \gamma_{3}, \quad \ldots, \quad \gamma_{l-2}, \gamma_{l-1}, \quad 0,0,0,0 \ldots\right)
$$

with $\gamma_{l-2}=\gamma_{l-1}=1$ and $l$ even or of the form

$$
(1,0,0,0, \ldots) \text {. }
$$

Example 1. The digital $(0,1)$-sequence over $\mathbb{Z}_{2}$ generated by

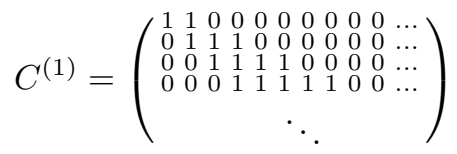

for example satisfies the conditions of Theorem 1 .

Example 2. The digital ( $\mathbf{T}, 2)$-sequence over $\mathbb{Z}_{2}$ generated by

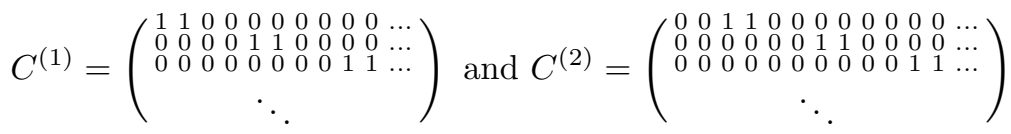

for example satisfies the conditions of Theorem 1 .

The proof of Theorem 1 will be given in Section 2 and is based on results on the distribution of the weighted sum-of-digits function $s_{q, \gamma}\left(n^{2}\right)$ of squares, represented in prime base $q$, for weight sequences $\gamma=\left(\gamma_{0}, \gamma_{1}, \gamma_{2}, \ldots\right)$ with $\gamma_{i} \in\{0,1, \ldots, q-1\}$ and with $\gamma_{i}=0$ for all $i$ large enough.

These results (together with some interesting corollaries) are given in Section 3 , In particular we compute in Section 3 ,

$$
\lim _{N \rightarrow \infty} \frac{\#\left\{0 \leq n<N \mid s_{q, \gamma}\left(n^{2}\right) \equiv d(\bmod q)\right\}}{N}
$$

(see Theorem 2).

In Section 4 we investigate the discrepancy of the subsequences $\left(\boldsymbol{x}_{n^{2}}\right)_{n>0}$ that are uniformly distributed. We obtain an upper bound for the star discrepancy that is based on the fascinating fact that we can identify a net structure in the subsequence. Indeed we will determine a function $\mathbf{T}^{\prime}: \mathbb{N} \rightarrow \mathbb{N}_{0}$ depending on the row lengths such that the subsequence $\left(\boldsymbol{x}_{n^{2}}\right)_{n>0}$ can be understood as a $\left(\mathbf{T}^{\prime}, s\right)$-sequence in base $q$. The class of $(\mathbf{T}, s)$-sequences in base $q$ represents a generalization of the digital $(\mathbf{T}, s)$-sequences over $\mathbb{Z}_{q}$. We call a sequence $\left(\boldsymbol{x}_{n}\right)_{n \geq 0}$ in $[0,1)^{s}$ a $(\mathbf{T}, s)$ sequence in base $q$ with quality parameter $\mathbf{T}: \mathbb{N} \rightarrow \mathbb{N}_{0}$ with $\mathbf{T}(m) \leq m$ if, for all integers $a \geq 0, m \geq 1$, the point set consisting of $\boldsymbol{x}_{n}$ with $a q^{m} \leq n<(a+1) q^{m}$ is a $(\mathbf{T}(m), m, s)$-net in base $q$; i.e., for every choice of $d_{1}, \ldots, d_{s} \geq 0$ satisfying $d_{1}+\cdots+d_{s}=m-\mathbf{T}(m)$ the point set is evenly distributed among all elementary intervals of order $\left(d_{1}, \ldots, d_{s}\right)$ that are intervals of the form

$$
I=\prod_{i=1}^{s}\left[\frac{a_{i}}{q^{d_{i}}}, \frac{a_{i}+1}{q^{d_{i}}}\right)
$$

with $a_{i} \in\left\{0, \ldots, q^{d_{i}}-1\right\}$. 
For a complete answer to the question concerning the uniform distribution of $\left(\boldsymbol{x}_{n^{2}}\right)_{n \geq 0}$ also for arbitrary digital $(\mathbf{T}, s)$-sequences we would need a generalization of the result of Theorem 2 to arbitrary (infinite) weight sequences, i.e., a generalization of the famous results of Mauduit and Rivat MR09 on the sum of digits of squares to weighted sums of digits. Several properties of the unweighted sum of digits of squares and also more general polynomial subsequences were also studied for example in Pet02, DR05, PW09.

\section{Proof of Theorem 1}

The proof of Theorem 1 follows immediately from the subsequent Proposition 1 and from the result of Theorem 2 (and of Corollary 1 respectively). Theorem 2 is formulated and proven in Section 3, so it remains to formulate and prove Proposition 1 in this section.

We consider a digital $(\mathbf{T}, s)$-sequence $\left(\boldsymbol{x}_{n}\right)_{n \geq 0}$ generated by the matrices $C^{(1)}$, $\ldots, C^{(s)}$ over $\mathbb{Z}_{q}$ with

$$
C^{(i)}:=\left(c_{j, k}^{(i)}\right)_{j, k \geq 0} .
$$

We denote the $j$ th row of $C^{(i)}$ by $c_{j}^{(i)}$. A necessary and sufficient condition for the uniform distribution of $\left(\boldsymbol{x}_{n}\right)_{n \geq 0}$ is that the rows of $C^{(1)}, \ldots, C^{(s)}$ altogether are linearly independent over $\mathbb{Z}_{q}$, i.e., that any finite set of rows of $C^{(1)}, \ldots, C^{(s)}$ is linearly independent over $\mathbb{Z}_{q}$.

If $\left(\boldsymbol{x}_{n}\right)_{n \geq 0}$ is not uniformly distributed, then it is not even dense; hence $\left(\boldsymbol{x}_{n^{2}}\right)_{n \geq 0}$ cannot be uniformly distributed. So in the following we can restrict to $C^{(1)}, \ldots, C^{(s)}$ with linearly independent rows.

Definition 2. We call a row $\gamma=\left(\gamma_{0}, \gamma_{1}, \ldots\right) \in \mathbb{Z}_{q}^{\mathbb{N}_{0}}$ of some $C^{(i)}$ admissible for $\left(n^{2}\right)_{n \geq 0}$ if

$$
\lim _{N \rightarrow \infty} \frac{1}{N} \#\left\{0 \leq n<N \mid s_{q, \gamma}\left(n^{2}\right) \equiv d(\bmod q)\right\}=\frac{1}{q}
$$

for all $d \in\{0,1, \ldots, q-1\}$.

Here by $s_{q, \gamma}(m)$ we denote the weighted sum-of-digits function of $m$; i.e.:

let $m=m_{0}+m_{1} q+\cdots+m_{r} q^{r}$ be the base $q$ representation of $m$; then

$$
s_{q, \gamma}(m):=\gamma_{0} m_{0}+\gamma_{1} m_{1}+\cdots+\gamma_{r} m_{r} .
$$

We show

Proposition 1. Let $\left(\boldsymbol{x}_{n}\right)_{n>0}$ be a digital $(\mathbf{T}, s)$-sequence over $\mathbb{Z}_{q}, q$ prime, generated by the matrices $C^{(1)}, \ldots, C^{(s)}$. Then the sequence $\left(\boldsymbol{x}_{n^{2}}\right)_{n \geq 0}$ is uniformly distributed if and only if every nontrivial linear combination of any finite set of rows of $C^{(1)}, \ldots, C^{(s)}$ over $\mathbb{Z}_{q}$ is admissible for $\left(n^{2}\right)_{n \geq 0}$.

Remark 1 . Note that this result is valid for arbitrary $C^{(1)}, \ldots, C^{(s)}$ and not only for matrices with finite rows exclusively. 
Proof of Proposition 1. Assume first that every nontrivial linear combination of any finite set of rows of $C^{(1)}, \ldots, C^{(s)}$ is admissible. To prove the uniform distribution of $\left(\boldsymbol{x}_{n^{2}}\right)_{n>0}$ it suffices to show that $\left(\boldsymbol{x}_{n^{2}}\right)_{n>0}$ is uniformly distributed in intervals of the form

$$
I:=\prod_{i=1}^{s}\left[\frac{b_{i}}{q^{d_{i}}}, \frac{b_{i}+1}{q^{d_{i}}}\right)
$$

with $d_{i} \geq 1$ and $0 \leq b_{i}<q^{d_{i}}$.

Let

$$
b_{i}=b_{0}^{(i)} q^{d_{i}-1}+b_{1}^{(i)} q^{d_{i}-2}+\cdots+b_{d_{i}-2}^{(i)} q+b_{d_{i}-1}^{(i)},
$$

and for $n \in \mathbb{N}_{0}$ let

$$
n^{2}=a_{0}\left(n^{2}\right)+q \cdot a_{1}\left(n^{2}\right)+q^{2} \cdot a_{2}\left(n^{2}\right)+\cdots
$$

be the base $q$ representation of $n^{2}$.

Then (with $e(x):=e^{2 \pi i x}$ )

$$
\begin{aligned}
& \frac{1}{N} \#\left\{0 \leq n<N \mid \boldsymbol{x}_{n^{2}} \in I\right\} \\
& =\frac{1}{N} \sum_{n=0}^{N-1} \frac{1}{q^{d_{1}+\cdots+d_{s}}} \prod_{i=1}^{s} \prod_{l_{i}=0}^{d_{i}-1} \sum_{u_{l_{i}}^{(i)}=0}^{q-1} e\left(\frac{u_{l_{i}}^{(i)}\left(c_{l_{i}, 0}^{(i)} a_{0}\left(n^{2}\right)+c_{l_{i}, 1}^{(i)} a_{1}\left(n^{2}\right)+\cdots-b_{l_{i}}^{(i)}\right)}{q}\right) \\
& =\frac{1}{q^{d_{1}+\cdots+d_{s}}}+\sum_{\substack{u_{l_{i}}^{(i)}=0 \\
1 \leq i \leq s \\
0 \leq l_{i} \leq d_{i}-1}}^{q-1} \frac{1}{q^{d_{1}+\cdots+d_{s}}} \cdot e\left(-\frac{1}{q} \sum_{i=1}^{s} \sum_{l_{i}=0}^{d_{i}-1} u_{l_{i}}^{(i)} b_{l_{i}}^{(i)}\right) \times \\
& \text { not all } u_{l_{i}}^{(i)}=0 \\
& \times \frac{1}{N} \sum_{n=0}^{N-1} e\left(\frac{1}{q}\left(a_{0}\left(n^{2}\right)\left(\sum_{i=1}^{s} \sum_{l_{i}=0}^{d_{i}-1} c_{l_{i}, 0}^{(i)} u_{l_{i}}^{(i)}\right)+a_{1}\left(n^{2}\right)\left(\sum_{i=1}^{s} \sum_{l_{i}=0}^{d_{i}-1} c_{l_{i}, 1}^{(i)} u_{l_{i}}^{(i)}\right)+\ldots\right)\right) .
\end{aligned}
$$

Let

$$
\gamma_{k}\left(\left(u_{l_{i}}^{(i)}\right)\right):=\sum_{i=1}^{s} \sum_{l_{i}=0}^{d_{i}-1} c_{l_{i}, k}^{(i)} u_{l_{i}}^{(i)}
$$

Then

$$
\gamma\left(\left(u_{l_{i}}^{(i)}\right)\right):=\left(\gamma_{0}\left(\left(u_{l_{i}}^{(i)}\right)\right), \gamma_{1}\left(\left(u_{l_{i}}^{(i)}\right)\right), \ldots\right)
$$

is a nontrivial linear combination of finitely many rows of $C^{(1)}, \ldots, C^{(s)}$ and different from $(0,0,0, \ldots)$; hence it is admissible for any choice of $\left(u_{l_{i}}^{(i)}\right)$, and consequently

$$
\lim _{N \rightarrow \infty} \frac{1}{N} \sum_{n=0}^{N-1} e\left(\frac{1}{q} \cdot s_{q, \gamma\left(\left(u_{l_{i}}^{(i)}\right)\right)}\left(n^{2}\right)\right)=0
$$

for all choices of $\left(u_{l_{i}}^{(i)}\right)$. So

$$
\lim _{N \rightarrow \infty} \frac{1}{N} \#\left\{0 \leq n<N \mid \boldsymbol{x}_{n^{2}} \in I\right\}=\frac{1}{q^{d_{1}+\cdots+d_{s}}}=\lambda(I),
$$

and the uniform distribution of $\left(\boldsymbol{x}_{n^{2}}\right)_{n \geq 0}$ follows. 
To show the "only if" part of the proposition we assume that $\left(\boldsymbol{x}_{n^{2}}\right)_{n>0}$ is uniformly distributed. We show the "only if" part in the following for the special case $q=2$ only. The general case runs along quite the same arguments but needs much more technical notation.

Let

$$
c_{l_{j_{i}}^{(i)}}^{(i)}=\left(c_{l_{j_{i}}^{(i)}, r}^{(i)}\right)_{r \geq 0}, \quad j_{i} \in\left\{0, \ldots, d_{i}-1\right\}, \quad i \in\{1, \ldots, s\}
$$

be $m:=d_{1}+\cdots+d_{s}$ arbitrary different rows of $C^{(1)}, \ldots, C^{(s)} \cdot\left(l_{j_{i}}^{(i)}<l_{j_{i}^{\prime}}^{(i)}\right.$ for $j_{i}<$ $j_{i}^{\prime}$.) We will show by induction on $m$ that $\sum_{i=1}^{s} \sum_{j_{i}=0}^{d_{i}-1} c_{l_{j_{i}}^{(i)}}^{(i)}$ is admissible for $\left(n^{2}\right)_{n \geq 0}$.

First let $m=1$ and consider a single row $c=\left(\gamma_{0}, \gamma_{1}, \ldots\right)$, say the $d$ th row of (w.l.o.g.) $C^{(1)}$. Consider the subset

$$
\begin{aligned}
S=\bigcup_{b=0}^{2^{d}-1}\left[\frac{2 b}{2^{d+1}}, \frac{2 b+1}{2^{d+1}}\right) \times \prod_{l=2}^{s}[0,1) \\
=\left\{\left(y_{1}, \ldots, y_{s}\right) \in[0,1)^{s} \mid \text { the } d \text { th digit of } y_{1} \text { equals } 0\right\} .
\end{aligned}
$$

By the assumption of uniform distribution on the one hand we have

$$
\lim _{N \rightarrow \infty} \frac{1}{N} \#\left\{0 \leq n<N \mid \boldsymbol{x}_{n^{2}} \in S\right\}=\frac{1}{2},
$$

and on the other hand,

$$
\begin{aligned}
\frac{1}{N} \#\left\{0 \leq n<N \mid \boldsymbol{x}_{n^{2}} \in S\right\} & =\frac{1}{N} \sum_{n=0}^{N-1} \frac{1}{2} \sum_{u=0}^{1}(-1)^{u \cdot\left(\gamma_{0} a_{0}\left(n^{2}\right)+\gamma_{1} a_{1}\left(n^{2}\right)+\ldots\right)} \\
& =\frac{1}{2}+\frac{1}{2 N} \sum_{n=0}^{N-1}(-1)^{\gamma_{0} a_{0}\left(n^{2}\right)+\gamma_{1} a_{1}\left(n^{2}\right)+\ldots}
\end{aligned}
$$

Hence $\frac{1}{2 N} \sum_{n=0}^{N-1}(-1)^{\gamma_{0} a_{0}\left(n^{2}\right)+\gamma_{1} a_{1}\left(n^{2}\right)+\ldots}$ tends to 0 as $N$ increases; i.e., $c=$ $\left(\gamma_{0}, \gamma_{1}, \ldots\right)$ is admissible.

For the induction step we consider the set

$$
\begin{aligned}
S:=\left\{\left(y_{1}, \ldots, y_{s}\right) \in[0,1)^{s} \mid \text { the } l_{j_{i}}^{(i)} \text { th digit of } y_{i} \text { equals } 0\right. \\
\left.\qquad \text { for all } j_{i} \in\left\{0, \ldots, d_{i}-1\right\}, i \in\{1, \ldots, s\}\right\} .
\end{aligned}
$$

$S$ is the union of finitely many intervals and has volume $2^{-m}$. Hence

$$
\lim _{N \rightarrow \infty} \frac{1}{N} \#\left\{0 \leq n<N \mid \boldsymbol{x}_{n^{2}} \in S\right\}=\frac{1}{2^{m}}
$$


On the other hand,

$$
\begin{aligned}
& \frac{1}{N} \#\left\{0 \leq n<N \mid \boldsymbol{x}_{n^{2}} \in S\right\} \\
& =\frac{1}{N} \sum_{n=0}^{N-1} \frac{1}{2^{m}} \prod_{i=1}^{s} \prod_{j_{i}=0}^{d_{i}-1} \sum_{u_{j_{i}}^{(i)}=0}^{1}(-1)^{u_{j_{i}}^{(i)} \cdot\left(c_{l_{j_{i}}^{(i)}, 0}^{(i)} \cdot a_{0}\left(n^{2}\right)+c_{l_{j_{i}}^{(i)}, 1}^{(i)} \cdot a_{1}\left(n^{2}\right)+\ldots\right)} \\
& =\frac{1}{2^{m}}
\end{aligned}
$$

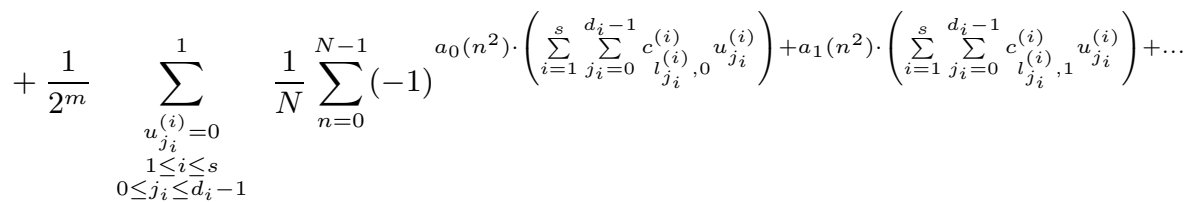

$$
\begin{aligned}
& \text { not all } u_{j_{i}}^{(i)}=0 \\
& \text { not all } u_{j_{i}}^{(i)}=1
\end{aligned}
$$

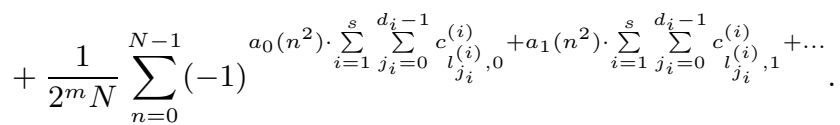

By the induction hypothesis the second summand of the above summands tends to zero; hence the last summand tends to zero and therefore

$$
\sum_{i=1}^{s} \sum_{j_{i}=0}^{d_{i}-1} c_{l_{j_{i}}^{(i)}}^{(i)}
$$

is admissible.

This completes the proof of Proposition 1 .

\section{ON THE Distribution OF THE Weighted SUM OF DIGITS OF SQUARES}

In this section we study for primes $q$ the quantity

$$
\begin{aligned}
T_{\gamma, d, q} & :=\lim _{N \rightarrow \infty} T_{\gamma, d, q}(N), \text { where } \\
T_{\gamma, d, q}(N) & :=\frac{1}{N} \cdot \#\left\{0 \leq n<N \mid s_{q, \gamma}\left(n^{2}\right) \equiv d(\bmod q)\right\}
\end{aligned}
$$

for finite weight sequences $\gamma=\left(\gamma_{0}, \gamma_{1}, \ldots, \gamma_{l-1}, 0,0, \ldots\right)$ with $d, \gamma_{i} \in\{0,1, \ldots, q-1\}$ and $\gamma_{l-1} \neq 0$. (Here and in the following $l \in \mathbb{N}$ is the minimal index such that $\gamma_{i}=0$ for all $i \geq l$.)

The main result is

\section{Theorem 2.}

(1) If $q=2$, then

- for l even:

$$
T_{\gamma, 0,2}= \begin{cases}\frac{1}{2} & \text { if } \gamma_{l-2}=1 \\ \frac{1}{2}+2^{-\frac{l}{2}} & \text { if } \gamma_{l-2}=0\end{cases}
$$


- for l odd:

$$
T_{\gamma, 0,2}= \begin{cases}\frac{1}{2} & \text { if } l=1 \\ \frac{1}{2}-2^{-\frac{l+1}{2}} & \text { if } l \geq 3 \text { and } \gamma_{l-3}=1, \\ \frac{1}{2}+2^{-\frac{l+1}{2}} & \text { if } l \geq 3 \text { and } \gamma_{l-3}=0 .\end{cases}
$$

(2) If $q \geq 3$, then let $C:=\left\{c_{1}, \ldots, c_{(q-1) / 2}\right\}$ be the set of the $c_{i}$ with $0 \leq c_{i}<q$ and $c_{i}^{(q-1) / 2} \equiv 1(\bmod q)$. Then

- for l even:

$$
T_{\gamma, d, q}=\frac{1}{q}\left(1-q^{-\frac{l}{2}}\right)+ \begin{cases}q^{-\frac{l}{2}} & \text { if } d=0, \\ 0 & \text { else. }\end{cases}
$$

- for l odd:

$$
T_{\gamma, d, q}=\frac{1}{q}\left(1-q^{\frac{1-l}{2}}\right)+ \begin{cases}q^{-\frac{1+l}{2}} & \text { if } d=0 \\ 2 q^{-\frac{1+l}{2}} & \text { if } d \in C, \\ 0 & \text { else. }\end{cases}
$$

We immediately obtain the following two corollaries.

Corollary 1. The finite weight sequence $\gamma=\left(\gamma_{0}, \gamma_{1}, \ldots, \gamma_{l-1}, 0,0, \ldots\right)$ with $\gamma_{l-1} \neq$ 0 is admissible for $\left(n^{2}\right)_{n \geq 0}$ if and only if $q=2, l$ even and $\gamma_{l-2}=\gamma_{l-1}=1$ or if $q=2, \gamma_{0}=1$ and $\gamma_{i}=0$ for $i \geq 1$.

Corollary 2. Let $Z_{k, d, q}$ be the frequency of the occurrence of digit $d$ as the $k$ th digit in the base $q$ representation of a square, i.e.,

$$
Z_{k, d, q}=\lim _{N \rightarrow \infty} \frac{1}{N} \cdot \#\left\{0 \leq n<N \mid n^{2}=a_{0}+a_{1} q+\cdots \text { with } a_{k}=d\right\} .
$$

(1) If $q=2$, then

$$
Z_{k, 0,2}= \begin{cases}\frac{1}{2} & \text { for } k=0 \\ \frac{1}{2}+2^{-\frac{k+1}{2}} & \text { for } k \text { odd } \\ \frac{1}{2}+2^{-\frac{k+2}{2}} & \text { else. }\end{cases}
$$

(2) If $q \geq 3$, then

$$
Z_{k, 0, q}= \begin{cases}\frac{1}{q}\left(1-q^{-\frac{k+1}{2}}\right)+q^{-\frac{k+1}{2}} & \text { for } k \text { odd }, \\ \frac{1}{q} & \text { for } k \text { even. }\end{cases}
$$

For $d \in C$ (as in Theorem 2) we have

$$
Z_{k, d, q}= \begin{cases}\frac{1}{q}\left(1-q^{-\frac{k+1}{2}}\right) & \text { for } k \text { odd } \\ \frac{1}{q}+q^{-\frac{k+2}{2}} & \text { for } k \text { even } .\end{cases}
$$

For $d \neq 0, d \notin C$ we have

$$
Z_{k, d, q}= \begin{cases}\frac{1}{q}\left(1-q^{-\frac{k+1}{2}}\right) & \text { for } k \text { odd } \\ \frac{1}{q}\left(1-q^{-\frac{k}{2}}\right) & \text { for } k \text { even }\end{cases}
$$


Remark 2. Prodinger and Wagner proved in [PW09, Theorem 9] an explicit formula for the so-called digit-generating function of powers $x^{v}, v \in \mathbb{N}$, in the binary case. Note that Corollary 2 gives the coefficients of the digit-generating function of the polynomial $p(x)=x^{2}$ in any prime base $q$ and for every digit $d$. For the special case where $q=v=2$ and $d=1$ the equality to the formula in [PW09, Theorem 9] can be easily checked.

Remark 3. Peter [Pet02, Proof of Lemma 2.1] studied for any base $q \geq 2$ the magnitude

$$
\#\left\{0 \leq n<q^{k+1} \mid d_{k}\left(n^{v}\right)=d\right\},
$$

which counts the occurrence of the digit $d$ as the $k$ th digit in the base $q$ representation of $n^{v}$ for fixed $v \in \mathbb{N}$. He deduced for (11) the following upper bound:

$$
q^{k}+\mathcal{O}\left(q^{k} 2^{-k /(2 v)}\right)
$$

This asymptotic result is valid for all powers $k \in \mathbb{N}$ and all bases $q$, but it does not distinguish between the different values of the digit $d$. Our Corollary 2 provides exact results for (11) for each choice of digit $d$ in prime base $q$ representation of squares.

For the proof of Theorem 2 we need the following lemmas.

Lemma 1. Let $L$ be the number of solutions of $x^{2} \equiv a\left(\bmod 2^{l}\right)$ with $x \in\{0,1, \ldots$, $\left.2^{l}-1\right\}$.

(1) If $l$ is even, then

$$
\begin{aligned}
& L=2^{\frac{l}{2}} \quad \text { for } a=0, \\
& L=2^{\frac{l}{2}} \quad \text { for } a=2^{l-2} \text {, } \\
& L=2^{v+2} \quad \text { for } a=2^{2 v} \cdot b \text { with } b \equiv 1(\bmod 8) \text { and } v \in\{0, \ldots,(l-4) / 2\} \text {, } \\
& L=0 \quad \text { otherwise. }
\end{aligned}
$$

(2) If $l$ is odd, then

$$
\begin{aligned}
& L=2^{\frac{l-1}{2}} \quad \text { for } a=0 \text {, } \\
& L=2^{\frac{l-1}{2}} \quad \text { for } a=2^{l-1} \text {, } \\
& L=2^{v+2} \quad \text { for } a=2^{2 v} \cdot b \text { with } b \equiv 1(\bmod 8) \text { and } v \in\{0, \ldots,(l-3) / 2\} \text {, } \\
& L=0 \quad \text { otherwise. }
\end{aligned}
$$

Lemma 2. Let $q$ be an odd prime and let $L$ be the number of solutions of $x^{2} \equiv$ $a\left(\bmod q^{l}\right)$ with $x \in\left\{0,1, \ldots, q^{l}-1\right\}$.

(1) If $l$ is even, then

$$
\begin{array}{ll}
L=q^{\frac{l}{2}} & \text { for } a=0, \\
L=2 q^{i} & \text { for } a=q^{2 i}(c+q w) \\
& \text { with } w \in\left\{0,1, \ldots, q^{l-2 i-1}-1\right\} \\
& \text { and } i \in\left\{0,1, \ldots, \frac{l}{2}-1\right\} \\
& \text { and } c \in C \text { (as in Theorem 2), } \\
L=0 & \text { otherwise. }
\end{array}
$$


(2) If $l$ is odd, then

$$
\begin{array}{ll}
L=q^{\frac{l-1}{2}} & \text { for } a=0, \\
L=2 q^{i} & \text { for } a=q^{2 i}(c+q w) \\
& \text { with } w \in\left\{0,1, \ldots, q^{l-2 i-1}-1\right\} \\
& \text { and } i \in\left\{0,1, \ldots, \frac{l+1}{2}-1\right\} \\
& \text { and } c \in C \text { (as in Theorem 2), } \\
L=0 & \text { otherwise. }
\end{array}
$$

The two lemmas follow from basic facts on quadratic congruences (e.g. from [HS79, p. 75ff.]). We omit the elementary proofs.

Proof of Theorem 2. Since for $n \equiv m\left(\bmod q^{l}\right)$ we have $s_{q, \gamma}\left(n^{2}\right)=s_{q, \gamma}\left(m^{2}\right)$, we obtain

$$
\left\lfloor\frac{N}{q^{l}}\right\rfloor \cdot q^{l} \cdot T_{\gamma, d, q}\left(q^{l}\right) \leq N \cdot T_{\gamma, d, q}(N) \leq\left(\left\lfloor\frac{N}{q^{l}}\right\rfloor+1\right) \cdot q^{l} \cdot T_{\gamma, d, q}\left(q^{l}\right) ;
$$

hence $T_{\gamma, d, q}=T_{\gamma, d, q}\left(q^{l}\right)$.

First, we prove Part (1):

Let $A_{0}:=A_{\gamma, 0, l}:=\left\{0 \leq a<2^{l} \mid s_{2, \gamma}(a) \equiv 0(\bmod 2)\right\}$.

- For $l$ even:

Then $0 \in A_{0}$, and $2^{l-2} \in A_{0}$ iff $\gamma_{l-2}=0$.

We further determine the number of $a \in A_{0}$ of the form

$$
a=2^{2 v} \cdot b \text { with } b \equiv 1(\bmod 8) \text { and } v \in\{0, \ldots, l / 2-2\},
$$

i.e.,

$$
a=0000 \ldots 00100 a_{2 v+3} \ldots a_{l-2} a_{l-1} .
$$

For $a_{2 v+3}, \ldots, a_{l-2}$ arbitrary the digit $a_{l-1}$ is uniquely determined by the condition $a \in A_{0}$. Hence the number of $a \in A_{0}$ of this form for given $v$ is $2^{l-2 v-4}$.

Hence by Lemma 1 we have

$$
\begin{aligned}
2^{l} \cdot T_{\gamma, 0,2}\left(2^{l}\right) & =2^{\frac{l}{2}}+\sum_{v=0}^{\frac{l}{2}-2} 2^{v+2} \cdot 2^{l-2 v-4}+ \begin{cases}0 & \text { if } \gamma_{l-2}=1, \\
2^{\frac{l}{2}} & \text { if } \gamma_{l-2}=0,\end{cases} \\
& = \begin{cases}2^{l-1} & \text { if } \gamma_{l-2}=1, \\
2^{l-1}+2^{\frac{l}{2}} & \text { if } \gamma_{l-2}=0 .\end{cases}
\end{aligned}
$$

- Now let $l$ be odd:

If $l=1$ we trivially have $T_{\gamma, 0,2}=1 / 2$.

Let $l \geq 3$. Then $0 \in A_{0}$ and $2^{l-1} \notin A_{0}$.

We determine the number of $a \in A_{0}$ of the form

$$
a=2^{2 v} \cdot b \text { with } b \equiv 1(\bmod 8) \text { and } v \in\{0, \ldots,(l-3) / 2\} .
$$


Here we have to handle the special case $v=(l-3) / 2$, i.e., $a=2^{l-3}$. For this $a$ we have $a \in A_{0}$ if and only if $\gamma_{l-3}=0$.

If $v \leq(l-5) / 2$, then by the same argument as in the case of $l$ even we have that the number of $a \in A_{0}$ of this form for given $v$ is $2^{l-2 v-4}$.

Hence by Lemma 1 we have

$$
\begin{aligned}
2^{l} \cdot T_{\gamma, 0,2}\left(2^{l}\right) & =2^{\frac{l-1}{2}}+\sum_{v=0}^{\frac{l-5}{2}} 2^{v+2} \cdot 2^{l-2 v-4}+ \begin{cases}0 & \text { if } \gamma_{l-3}=1, \\
2^{\frac{l+1}{2}} & \text { if } \gamma_{l-3}=0,\end{cases} \\
& = \begin{cases}2^{l-1}-2^{\frac{l-1}{2}} & \text { if } \gamma_{l-3}=1, \\
2^{l-1}+2^{\frac{l-1}{2}} & \text { if } \gamma_{l-3}=0 .\end{cases}
\end{aligned}
$$

The result follows.

Now we handle Part (2):

Let $A_{d}:=A_{\gamma, d, l}:=\left\{0 \leq a<q^{l} \mid s_{q, \gamma}(a) \equiv d(\bmod q)\right\}$.

- Consider $l$ even:

Trivially $0 \in A_{0}$ and therefore $0 \notin A_{d}$ for $d>0$.

We determine the number of $a \in A_{d}$ of the form

$a=q^{2 i}(c+q w)$ with $w \in\left\{0, \ldots, q^{l-2 i-1}-1\right\}, i \in\{0, \ldots, l / 2-1\}, c \in C$,

i.e.,

$a=00 \ldots 00 c a_{2 i+1} \ldots a_{l-2} a_{l-1}$.

By the same argument as in Part (11) the number of $a \in A_{d}$ is given by $q^{l-2 i-2}$ for $c$ and $i$ fixed.

Altogether by Lemma 2 we have

$$
\begin{aligned}
q^{l} \cdot T_{\gamma, d, q}\left(q^{l}\right) & =\frac{q-1}{2} \sum_{i=0}^{\frac{l}{2}-1} 2 q^{i} \cdot q^{l-2 i-2}+ \begin{cases}q^{\frac{l}{2}} & \text { if } d=0, \\
0 & \text { else },\end{cases} \\
& =q^{l-1}\left(1-q^{-\frac{l}{2}}\right)+ \begin{cases}q^{\frac{l}{2}} & \text { if } d=0, \\
0 & \text { else. }\end{cases}
\end{aligned}
$$

- Let $l$ be odd:

As in the case $l$ even we have $0 \in A_{0}, 0 \notin A_{d}$ for $d>0$.

We determine the number of $a \in A_{d}$ of the form $a=q^{2 i}(c+q w)$ with $w \in\left\{0, \ldots, q^{l-2 i-1}-1\right\}, i \in\{0, \ldots,(l-1) / 2\}, c \in C$.

In the case $i=(l-1) / 2$ we have $a=c q^{l-1}$; hence $a \in A_{d}$ if and only if $d \in C$.

If $i \leq(l-3) / 2$, by the same argument as in the case $l$ even, the number of $a \in A_{d}$ of this form is given by $q^{l-2 i-2}$ for $c$ and $i$ fixed. 
Hence by Lemma 2 we have

$$
\begin{aligned}
q^{l} \cdot T_{\gamma, d, q}\left(q^{l}\right) & =\frac{q-1}{2} \sum_{i=0}^{\frac{l-3}{2}} 2 q^{i} \cdot q^{l-2 i-2}+ \begin{cases}q^{\frac{l-1}{2}} & \text { if } d=0, \\
2 q^{\frac{l-1}{2}} & \text { if } d \in C, \\
0 & \text { else, }\end{cases} \\
& =q^{l-1}\left(1-q^{\frac{1-l}{2}}\right)+ \begin{cases}q^{\frac{l-1}{2}} & \text { if } d=0, \\
2 q^{\frac{l-1}{2}} & \text { if } d \in C, \\
0 & \text { else. }\end{cases}
\end{aligned}
$$

This completes the proof of Theorem 2 ,

\section{ON THE DISCREPANCY OF THE UNIFORMLY DISTRIBUTED QUADRATIC SUBSEQUENCES}

For the investigation of the discrepancy we make use of the function $L: \mathbb{N}_{0}^{s} \rightarrow \mathbb{N}_{0}$ that measures the lengths of the matrix rows as follows:

$$
\left(d_{1}, \ldots, d_{s}\right) \mapsto \max _{1 \leq i \leq s} \max _{0 \leq j<d_{i}}\left(\max \left\{k \in \mathbb{N}_{0} \mid c_{j, k}^{(i)} \neq 0\right\}+1\right),
$$

where the maximum of the empty set is defined as 0 here and later on.

Theorem 3. Let $\left(\boldsymbol{x}_{n^{2}}\right)_{n \geq 0}$ be a uniformly distributed subsequence of a digital $(\mathbf{T}, s)$-sequence over $\mathbb{Z}_{2}$ generated by matrices of finite rows. Then $\left(\boldsymbol{x}_{n^{2}}\right)_{n \geq 0}$ is a $\left(\mathbf{T}^{\prime}, s\right)$-sequence in base 2 with $\mathbf{T}^{\prime}: \mathbb{N} \rightarrow \mathbb{N}_{0}$ defined by

$$
\mathbf{T}^{\prime}(m)=m-\min _{1 \leq i \leq s} \max \{d \in \mathbb{N}: L(\underbrace{0, \ldots, 0}_{i-1}, d, 0, \ldots, 0) \leq m\} .
$$

Therefore its star discrepancy satisfies for all $N \in \mathbb{N}$ the well-known discrepancy bound (see e.g. [DP10, Theorem 5.16])

$$
\begin{aligned}
N D_{N}^{*} \leq & \sum_{i=0}^{s-1} \sum_{m=n}^{r+1} 2^{\mathbf{T}^{\prime}(m)-1}\left(\begin{array}{c}
m-\mathbf{T}^{\prime}(m) \\
i
\end{array}\right) \\
& +2^{\mathbf{T}^{\prime}(n)-1} \sum_{i=0}^{s-1}\left(\begin{array}{c}
n-\mathbf{T}^{\prime}(n) \\
i
\end{array}\right),
\end{aligned}
$$

where $r \in \mathbb{N}_{0}$ such that $2^{r} \leq N<2^{r+1}$ and $n$ is the largest integer satisfying $2^{n} \mid N$.

Proof. We fix an $m \in \mathbb{N}$ and prove for any consecutive $2^{m}$ points of the subsequence that they form a $\left(\mathbf{T}^{\prime}(m), m, s\right)$-net in base 2 . We choose nonnegative integers $d_{1}, \ldots, d_{s}$ such that $d_{1}+\cdots+d_{s}=m-\mathbf{T}^{\prime}(m)$ and consider all elementary intervals $I$ of order $\left(d_{1}, \ldots, d_{s}\right)$. From the definition of $\mathbf{T}^{\prime}$ we derive

$$
d_{1}+\cdots+d_{s}=\min _{1 \leq i \leq s} \max \{d \in \mathbb{N}: L(\underbrace{0, \ldots, 0}_{i-1}, d, 0, \ldots, 0) \leq m\}
$$

and therefore

$$
d_{j} \leq \min _{1 \leq i \leq s} \max \{d \in \mathbb{N}: L(\underbrace{0, \ldots, 0}_{i-1}, d, 0, \ldots, 0) \leq m\}
$$

and $L\left(0, \ldots, 0, d_{j}, 0, \ldots, 0\right) \leq m$ for every $j \in\{1, \ldots, s\}$. Hence $L\left(d_{1}, \ldots, d_{s}\right) \leq m$. This yields that the residue of $n^{2}$ modulo $2^{m}$ decides whether $\boldsymbol{x}_{n^{2}}$ is included in $I$ or not. Now the assumption that $\boldsymbol{x}_{n^{2}}$ is uniformly distributed together with the 
fact that $\left(n+2^{m}\right)^{2} \equiv n^{2}\left(\bmod 2^{m}\right)$ implies that any consecutive $2^{m}$ points of the subsequence form a $\left(\mathbf{T}^{\prime}(m), m, s\right)$-net.

Example 3. We consider the digital $(0,1)$-sequence over $\mathbb{Z}_{2}$ of Example 1 that is generated by the following matrix:

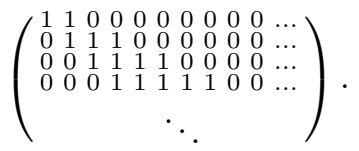

Obviously we have $L(d)=2 d$ and therefore the subsequence indexed by the squares is a $\left(\mathbf{T}^{\prime}, 1\right)$-sequence in base 2 with $\mathbf{T}^{\prime}(m)=m-\max \{d \in \mathbb{N}: 2 d \leq m\}=$ $m-\lfloor m / 2\rfloor=\lfloor(m+1) / 2\rfloor$. Its star discrepancy satisfies for all $N \in \mathbb{N}$,

$$
\begin{aligned}
N D_{N}^{*} & \leq \frac{1}{2}\left(1+\sum_{m=0}^{\left\lfloor\log _{2}(N)\right\rfloor+1} 2^{\mathbf{T}^{\prime}(m)}\right) \\
& \leq \frac{1}{2}\left(1+\sum_{m=0}^{\left\lfloor\left(\left\lfloor\log _{2}(N)\right\rfloor+1\right) / 2\right\rfloor}\left(2^{\mathbf{T}^{\prime}(2 m)}+2^{\mathbf{T}^{\prime}(2 m+1)}\right)\right) \\
& \leq \frac{1}{2}\left(1+\sum_{m=0}^{\left\lfloor\left(\left\lfloor\log _{2}(N)\right\rfloor+1\right) / 2\right\rfloor}\left(2^{m}+2^{m+1}\right)\right)=\mathcal{O}\left(2^{\log _{2}(N) / 2}\right)=\mathcal{O}(\sqrt{N})
\end{aligned}
$$

with an implied constant that does not depend on $N$.

Example 4. The star discrepancy of the subsequence indexed by the squares of the digital $(\mathbf{T}, 2)$-sequence over $\mathbb{Z}_{2}$ generated by

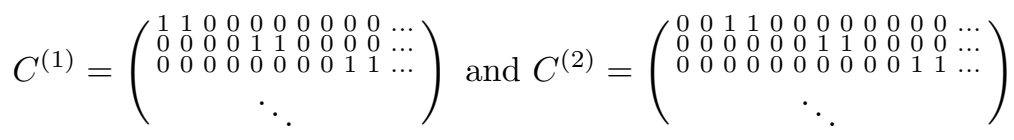

satisfies

$$
N D_{N}^{*}=\mathcal{O}\left(\log (N) N^{3 / 4}\right)
$$

with an implied constant that does not depend on $N$. From $L(d, 0)=4 d-2$ and $L(0, d)=4 d$ we derive $\mathbf{T}^{\prime}(m)=m-\lfloor m / 4\rfloor$. Now the upper bound in Theorem 3 and similar argumentations as in the example above yield

$$
N D_{N}^{*}=\mathcal{O}\left(\sum_{m=0}^{\left\lfloor\log _{2}(N)\right\rfloor+1}(1+\lfloor m / 4\rfloor) 2^{m-\lfloor m / 4\rfloor}\right)=\mathcal{O}\left(\log (N) N^{3 / 4}\right) .
$$

From Example 3 and Example 4 it is clear that in the $s$-dimensional case one can find generator matrices of a digital $(\mathbf{T}, s)$-sequence over $\mathbb{Z}_{2}$ such that the subsequence indexed by the squares satisfies

$$
N D_{N}^{*}=\mathcal{O}\left(\log ^{s-1}(N) N^{\frac{2 s-1}{2 s}}\right) .
$$

Remark 4. The method of proof used for Theorem 3 can be generalized for more arbitrary polynomials $p(x) \in \mathbb{Z}[x]$. Nevertheless in order to apply such a generalized discrepancy bound we need a criterion to decide the uniformity of the subsequence in the style of the one in Theorem 1 For monomials in the binary case one could 
use the coefficients of the digit-generating function given in [PW09, Theorem 9] to determine some admissible rows. For a classification of all admissible rows for an arbitrary prime base we would need results in the style of the ones in Lemmas 1 and 2 for congruences of the form $x^{v} \equiv a\left(\bmod q^{l}\right)$. Such solutions of such congruences are less studied than the quadratic ones. But with much more effort it seems to be possible to get information on the uniformity of, for example, cubic subsequences or other special choices of $v$. Of course such subsequences and also more arbitrary polynomial subsequences of digital $(\mathbf{T}, s)$-sequences are interesting for future research.

\section{ACKNOWLEDGEMENT}

The authors would like to thank the anonymous referee for bringing to their attention the articles Pet02, PW09, which contain interesting results that relate to the ones given in Corollary 2 ,

\section{REFERENCES}

[DP10] J. Dick and F. Pillichshammer. Digital Nets and Sequences. Discrepancy Theory and quasi-Monte Carlo Integration. Cambridge University Press, Cambridge, 2010. MR2683394

[DR05] M. Drmota and J. Rivat. The sum-of-digits function of squares. J. London Math. Soc. (2), 72:273-292, 2005. MR2156654 (2006d:11113)

[DT97] M. Drmota and R. Tichy. Sequences, discrepancies and applications, volume 1651 of Lecture Notes in Mathematics. Springer-Verlag, Berlin, 1997. MR:1470456 (98j:11057)

[Erd46] P. Erdös. On the distribution function of additive functions. Ann. of Math. (2), 47:120, 1946. MR0015424(7:416c)

[HKLP09] R. Hofer, P. Kritzer, G. Larcher, and F. Pillichshammer. Distribution properties of generalized van der Corput-Halton sequences and their subsequences. Int. J. Number Theory, 5(4):719-746, 2009. MR2532267(2010d:11082)

[HL10] R. Hofer and G. Larcher. On existence and discrepancy of certain digital NiederreiterHalton sequences. Acta Arith., 141(4):369-394, 2010. MR2587294 (2011b:11108)

[Hof09] R. Hofer. On subsequences of Niederreiter-Halton sequences. In Monte Carlo and Quasi-Monte Carlo Methods 2008, P. L'Ecuyer, B. Owen (eds.), Springer, Berlin, 2009, pp. 423-438. MR2743911(2011k:65008)

[HS79] E. Hlawka and J. Schoissengeier. Zahlentheorie Eine Einführung. Vorlesungen über Mathematik. Manzsche Verlags- und Universitätsbuchhandlung, Vienna, 1979. MR:548868(82d:10001)

[KN74] L. Kuipers and H. Niederreiter. Uniform distribution of sequences. Pure and Applied Mathematics. Wiley-Interscience [John Wiley \& Sons], New York, 1974. MR0419394 $(54: 7415)$

[LN95] G. Larcher and H. Niederreiter. Generalized $(t, s)$-sequences, Kronecker-type sequences, and Diophantine approximations of formal Laurent series. Trans. Amer. Math. Soc., 347(6):2051-2073, 1995. MR1290724(95i:11086)

[MR09] C. Mauduit and J. Rivat. La somme des chiffres des carrés. Acta Math., 203(1):107-148, 2009. MR2545827(2010j:11119)

[Nie92] H. Niederreiter. Random number generation and quasi-Monte Carlo methods, volume 63 of CBMS-NSF Regional Conference Series in Applied Mathematics. Society for Industrial and Applied Mathematics (SIAM), Philadelphia, PA, 1992. MR.1172997 (93h:65008)

[Pet02] M. Peter. The summatory function of the sum-of-digits function on polynomial sequences. Acta Arith., 104:85-96, 2002. MR1913736 (2003i:11140)

[PW09] H. Prodinger and S. Wagner. The digit generating function of a polynomial. J. Number Theory, 129:1325-1337, 2009. MR2521476 (2010g:11126)

[Vin48] I. M. Vinogradov. On an estimate of trigonometric sums with prime numbers (Russian). Izvestiya Akad. Nauk. SSSR. Ser. Mat., 12:225-248, 1948. MR0029418(10:599b) 
Institute of Financial Mathematics, University of Linz, Altenbergerstr. 69, 4040 Linz, Austria

E-mail address: roswitha.hofer@jku.at

Institute of Financial Mathematics, University of Linz, Altenbergerstr. 69, 4040 Linz, Austria

E-mail address: gerhard.larcher@jku.at

Institute of Financial Mathematics, University of Linz, Altenbergerstr. 69, 4040 LinZ, Austria

E-mail address: heidrun.zellinger@jku.at 